(C) 2006 IEEE. Personal use of this material is permitted. Permission from IEEE must be obtained for all other uses, in any current or future media, including reprinting/republishing this material for advertising or promotional purposes, creating new collective works, for resale or redistribution to servers or lists, or reuse of any copyrighted component of this work in other works. 


\title{
SNR Gap Approximation for M-PSK-Based Bit Loading
}

\author{
Ana García-Armada, Member, IEEE
}

\begin{abstract}
Adaptive OFDM has the potential of providing bandwidth-efficient communications in hostile propagation environments. Currently, bit loading algorithms use M-ary quadrature amplitude modulation of the OFDM sub-carriers, where the number of bits per symbol modulating each of them is obtained in order to maximize the performance. SNR gap approximation for M-QAM signaling makes the algorithms simpler to implement. However, in some circumstances it may be preferable to use M-ary phase shift keying. In this letter an approximation is derived for M-PSK similar to the SNR gap of M-QAM so that bit loading algorithms can be extended to this type of modulation. In addition, the performance obtained when using M-PSK is compared to that of M-QAM in a practical situation.
\end{abstract}

Index Terms-OFDM, bit loading, SNR gap.

\section{INTRODUCTION}

$\mathbf{O}$ FDM (Orthogonal Frequency Division Multiplexing) has been adopted for many wireless standards (e.g. DVB-T [1] for broadcasting, IEEE 802.11a [2] for WLAN) and is being investigated as a candidate technique for future broadband multimedia communications. Together with its well-known behavior in frequency-selective channels, this type of modulation has been traditionally used in wire-line communications because of its ability to approach channel capacity when combined with bit loading techniques [3]-[8]. In recent literature [9]-[11] several adaptive approaches using OFDM are targeting wireless systems with the aim of maximizing transmission rate.

Current bit loading algorithms use M-ary quadrature amplitude modulation (M-QAM) of the OFDM sub-carriers, where the number of bits per symbol modulating each of them is provided by the algorithm so that either transmitted energy is minimized, or overall throughput (bit rate) or energy margin are maximized, subject to a pre-defined error rate constraint. The use of M-QAM is motivated by the fact that it is more energy-efficient than M-ary phase shift keying (M-PSK) while retaining the same bandwidth-efficiency. Additionally, it is possible to use the SNR gap approximation for M-QAM [12], so the algorithms become simpler to implement [5], [9], [11].

However, the use of M-PSK may have other advantages. For example, some techniques that are used in OFDM in order to reduce its Peak-to-Average-power Ratio work only with M-PSK modulation [13], [14]. Also, the existing SNR gap approximation is accurate only for rectangular M-QAM constellations with constellation size equal to a power of 2 . The aim of this letter is to provide such an expression so as to extend bit loading algorithms to M-PSK-modulated OFDM.

\section{SNR GAP APPROXIMATION FOR M-PSK-BASED BIT LOADING}

OFDM divides the available bandwidth into a set of $N$ orthogonal sub-channels. With the insertion of a sufficiently long cyclic prefix, these $N$ sub-channels can be treated as independent parallel locally-flat channels corrupted by AWGN [4].

Most bit loading algorithms impose the condition that a given performance should be achieved in every sub-channel. Let us denote by $\gamma$ the required $\operatorname{SNR}\left(E_{s} / N_{0}\right)$ to achieve the target error probability in a given AWGN sub-channel when it carries $R=\log _{2}(M)$ bits per symbol either QAM- or PSK-modulated. SNR gap is used in order to relate $R$ and $\gamma$ in a straightforward manner, simplifying bit loading algorithms.

\section{A. Review of SNR gap for M-QAM}

When M-QAM modulation is used and error probability is measured in terms of SER (Symbol Error Rate), the number of bits per symbol $R$ may be found as [12]:

$$
R=\log _{2}\left(1+\frac{\gamma}{\Gamma}\right)
$$

with the SNR gap [4] defined as:

$$
\Gamma=\frac{1}{3}\left[Q^{-1}\left(\frac{S E R}{4}\right)\right]^{2},
$$

where $Q$ function is defined as:

$$
Q(x)=\int_{x}^{\infty} \frac{e^{-u^{2} / 2}}{\sqrt{2 \pi}} d u .
$$

The SNR gap is used to measure the reduction of SNR with respect to capacity and it only depends on the error probability requirements. The expression of (1) is accurate for rectangular constellations with size equal to a power of 2 and $R>1$. Although it is not always exact, it constitutes a good approximation that simplifies bit loading algorithms [5], [9], [11].

\section{B. SNR gap for M-PSK}

In order to find a similar definition for M-PSK modulation, let us recall that the relationship between error probability and SNR can be approximated by [15]:

$$
S E R \approx 2 Q\left(\sqrt{2 \gamma} \sin \left(\frac{\pi}{2^{R}}\right)\right) .
$$


TABLE I

EXAMPLE VALUES OF THE TERMS OF INEQUALITY (8) FOR BPSK AND QPSK

\begin{tabular}{cccc}
\hline SER & $\pi^{2} \cdot \Gamma^{*}(\mathrm{~dB})$ & $\gamma(\mathrm{dB})$ BPSK & $\gamma(\mathrm{dB})$ QPSK \\
\hline $10^{-3}$ & 7.3 & 6.8 & 10.3 \\
$10^{-4}$ & 8.8 & 8.4 & 11.8 \\
$10^{-5}$ & 9.9 & 9.6 & 12.9 \\
$10^{-6}$ & 10.8 & 10.5 & 13.8 \\
\hline
\end{tabular}

This approximation is very close to the exact values even for a small number of bits per symbol $(R)$ and it is more accurate than the one used to obtain SNR gap for M-QAM. However, the relationship between the modulation order and SNR is not linear, so that an expression similar to (1) does not apply to M-PSK. Therefore the definition of an SNR gap cannot be stated in the same way. Nevertheless, in order to simplify bit loading it may suffice with a closed-form expression relating $R$ and $\gamma$ that is true for all $M$.

From (4) $M$ may be approximately expressed as:

$$
M=\frac{\pi}{\sin ^{-1}\left(\frac{Q^{-1}(S E R / 2)}{(2 \gamma)^{1 / 2}}\right)} .
$$

If the argument of $\sin ^{-1}$ is small the approximation $\sin (x) \approx x$ can be used, so that $R$ may be written as:

$$
R=\frac{1}{2} \log _{2}\left(\frac{\gamma}{\Gamma^{*}}\right),
$$

with a modified $\Gamma^{*}$ defined as:

$$
\Gamma^{*}=\left(\frac{Q^{-1}(S E R / 2)}{\sqrt{2} \pi}\right)^{2} .
$$

Expressions (6) and (7) hold if the small angle approximation is valid, that is, if:

$$
\frac{Q^{-1}(S E R / 2)}{(2 \gamma)^{1 / 2}} \ll 1 \Rightarrow \pi^{2} \cdot \Gamma^{*} \ll \gamma .
$$

In order to check the validity of the approximation, it is interesting to note that the product $\pi^{2} \cdot \Gamma^{*}$ equals the SNR needed in BPSK to achieve a given error probability, since from (4):

$$
\gamma_{B P S K} \approx \pi^{2}\left(\frac{Q^{-1}(S E R / 2)}{\sqrt{2} \pi}\right)^{2}=\pi^{2} \cdot \Gamma^{*}
$$

This means that, even though the approximation is not accurate for BPSK, it will always be valid for modulations of higher order than BPSK, since the required SNR for $M>2$ will always be greater than the (approximated) value required for $M=2$, thus satisfying (8).

Table I compares the values of inequality (8) for several error probabilities, where $\gamma$ has been obtained for BPSK and QPSK using the exact expressions of [15]. We can see that $\gamma$ is $3 \mathrm{~dB}$ higher than $\pi^{2} \cdot \Gamma^{*}$ for QPSK. Obviously, the approximation is better for high SNR and high number of bits per symbol. It should be noted that when the condition (8) is satisfied, then the number of bits per symbol in (6) is always equal or greater than zero, as it should be.
Equations (6) and (7) constitute an approximation that relates $R$ and $\gamma$ and can therefore be used in bit loading algorithms. Table II summarizes approximations for M-QAM and M-PSK.

It should be pointed out that, although it is useful for bit loading, the modified $\Gamma^{*}$ for M-PSK does not have the same meaning as SNR gap for M-QAM. It is also possible to define an SNR gap with the same meaning of reduction of SNR with respect to capacity for M-PSK. However, this gap definition is dependent on SNR, which is not convenient for bit loading algorithms.

It is easy to verify that if we define $\Gamma$ for M-PSK as:

$$
\Gamma=\frac{\sqrt{\gamma \cdot \Gamma^{*}}}{1-\sqrt{\Gamma^{*} / \gamma}}
$$

then $\Gamma$ satisfies (1). Although not useful for our application, it is included for completeness (the development of (10) is provided as an appendix).

\section{M-PSK-BASED BIT LOADING}

Many algorithms can be found in the literature [5], [9], [11] that make use of the SNR gap approximation for M-QAM-based bit loading. All of them can be adapted to M-PSK by replacing expressions (1) and (2) with the equivalent developed expressions (6) and (7).

For ease of implementation, the number of bits per symbol allocated to each sub-channel is generally constrained to be of finite granularity while expressions (1) and (6) may produce non-integer values. Several approaches solve this problem: algorithm [7] is optimized constraining the number of bits per symbol in each sub-channel to be an integer and gives the optimum but complex solution which is not water-filling; algorithm [5] computes a bit distribution using SNR gap by rounding of approximate water-filling results, which is sub-optimum but efficient.

In order to compare the performance obtained in a practical situation, let us consider the two-user algorithm of [11] when M-PSK is used instead of M-QAM. In this algorithm, any user that wishes to transmit senses the channel and performs a constant-energy bit loading algorithm in free sub-channels so as to achieve the required bit rate with the minimum number of sub-channels while fulfilling a constraint on SER. The first user is able to choose among the 64 sub-carriers of an OFDM signal while the second user is only allowed to transmit in those frequencies that are not chosen by the first user. Signals transmitted by each user experience a different multipath channel according to Hiperlan model type A. The numbers of bits per symbol are computed using the SNR gap and are then rounded to nearest integer values as in [5]. More details about the algorithm and simulations may be found in [11], [16].

One of the advantages of using M-PSK instead of M-QAM for OFDM is the availability of techniques to reduce the signal's Peak-to-Average-power Ratio (PAR), also known as PAPR or PMEPR (Peak-to-Mean Envelope Power Ratio). Some of the techniques that offer the highest reductions work only with M-PSK modulation [13], [14]. For example, PAR can be reduced by $5-6 \mathrm{~dB}$ independently of the number of 
TABLE II

SNR GAP APPROXIMATIONS FOR M-QAM AND M-PSK

\begin{tabular}{|c|c|c|c|}
\hline M-QAM & $R=\log _{2} 1+\frac{\gamma}{\Gamma}$ & $\Gamma=\frac{1}{3}\left[Q^{-1} \frac{S E R}{4}\right]^{2}$ & Useful for bit loading \\
\hline M-PSK & $R=\frac{1}{2} \log _{2} \frac{\gamma}{\Gamma^{*}}$ & $\Gamma^{*}=\frac{Q^{-1}(S E R / 2)}{\sqrt{2} \pi}$ & Useful for bit loading \\
\hline M-PSK & $R=\log _{2} 1+\frac{\gamma}{\Gamma}$ & $\Gamma=\frac{\sqrt{\gamma \cdot \Gamma^{*}}}{1-\sqrt{\Gamma^{*} / \gamma}}$ & Not useful for bit loading \\
\hline
\end{tabular}

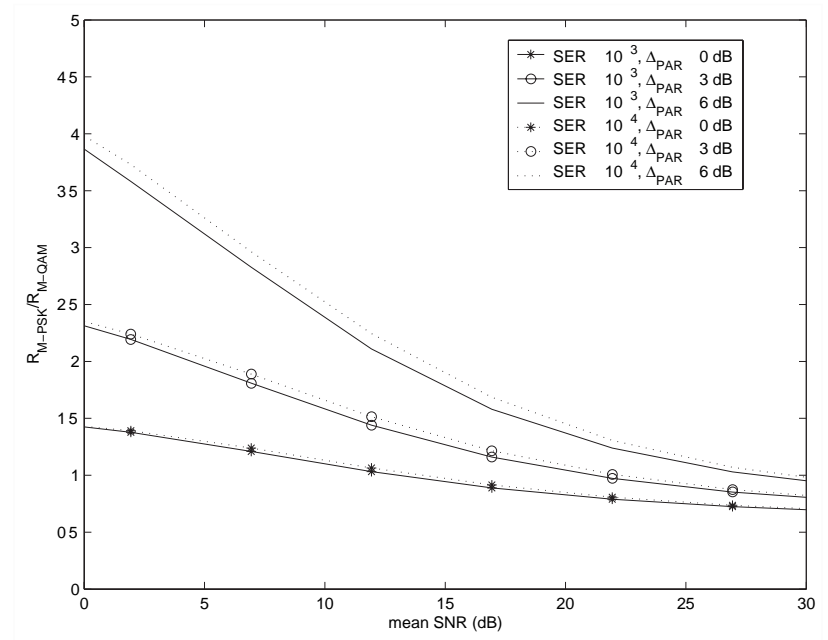

Fig. 1. Ratio of the number of bits per symbol achieved with both modulation schemes: user 1.

sub-carriers with the scheme of [14] only for M-PSK whereas reduction schemes that work with any type of modulation obtain reductions on the order of $1-4 \mathrm{~dB}$ as reported in the literature [17], [18]. Therefore, it is interesting to compare the performance of bit loading algorithms based in M-PSK and M-QAM with the transmitted signal constrained to the same PAR. Given that PAR reduction techniques are not equally powerful for both schemes, the power available for transmission must be lowered for M-QAM if the signals are to be subject to the same degree of non-linear effects as a result of amplification. In the following we denote this reduction by $\triangle_{P A R}$.

The ratios of the number of bits per symbol achieved with the algorithm when using M-PSK and M-QAM are shown in Fig. 1 and Fig. 2 for user 1 and 2 respectively. Each value has been obtained by simulation, allocating to each user the highest possible number of bits per symbol to achieve a target error probability $\left(S E R \leq 10^{-3}\right.$ or $\left.10^{-4}\right)$ and averaging over 1000 channel realizations. Mean SNR is defined for each user as:

$$
\text { mean } S N R=\frac{1}{N_{u}} \sum_{n=0}^{N_{u}-1} \gamma_{n}
$$

with $N_{u}$ equal to the number of sub-channels in which transmission is carried out and $\gamma_{n}$ denoting the SNR in $n$-th sub-channel.

M-PSK-based bit loading produces a higher total number

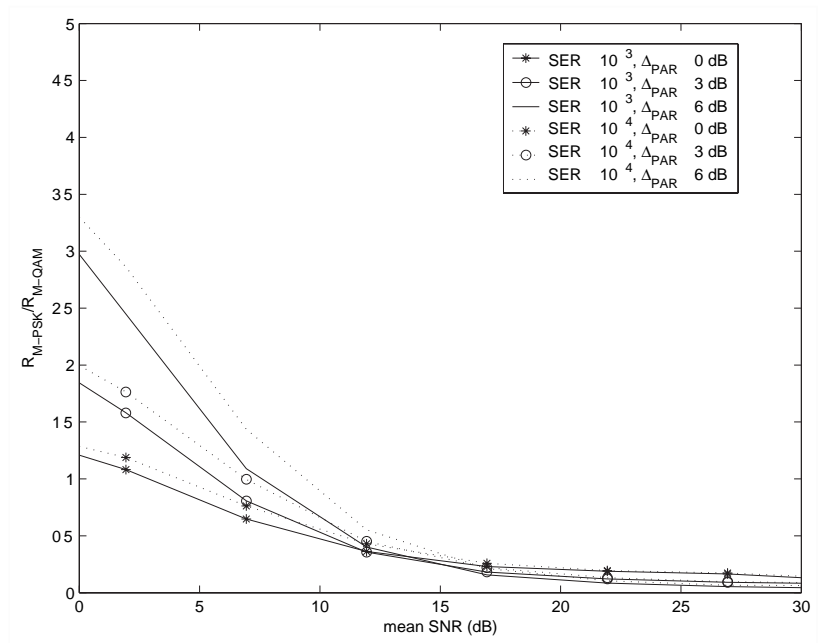

Fig. 2. Ratio of the number of bits per symbol achieved with both modulation schemes: user 2.

of bits per symbol than M-QAM because the expressions (1) and (2) for M-QAM underestimate the value of $R$ if SNR and target SER are low ${ }^{1}$. When SNR is high, then M-QAM is able to allocate more bits per symbol and the performance of M-QAM-based bit loading is better. However, as we can see from inspection of Fig. 1, mean SNR must be very high in order for M-QAM to perform better when we consider the first user. For mean SNR values ranging from 0 to $30 \mathrm{~dB}$, the algorithm using expressions (6) and (7) achieves a higher number of bits per symbol.

It is interesting to examine the different behavior of the two users depending on the variability of SNR in the sub-channels after the algorithm allocates bits and energy to them. The loaded sub-carriers of user 1 have small SNR variability so that when mean SNR is low, SNR in most sub-channels is low and consequently M-PSK performs better than M-QAM. However, for user 2 (which finds many of the sub-channels unavailable) the SNR variability is higher ${ }^{2}$. The result of the algorithm for this user is such that some sub-channels with very high SNR are loaded with a high number of bits per symbol and a much more reduced number of sub-channels with low SNR are assigned smaller loads (if loaded at all). In this case,

${ }^{1}$ The actual SER would be smaller for M-QAM than for M-PSK while both of them fullfilling the required SER.

${ }^{2}$ As an example, we found that the standard deviation of the SNR per sub-channel of user 2 is $5 \mathrm{~dB}$ higher than the standard deviation of user 1 for the same mean SNR value of $7 \mathrm{~dB}$ when running the M-PSK algorithm for a target SER of $10^{-3}$. 
highly loaded sub-channels are dominant and determine the performance so that even for small mean SNR, the difference between M-QAM and M-PSK is not so significant.

Finally it can be seen that the performance of M-PSK with respect to M-QAM improves (up to more than two times) when we consider the difference in PAR reduction capabilities $\left(\Delta_{P A R}\right)$ between them.

\section{CONCLUSIONS}

A modified SNR gap approximation has been derived for M-PSK modulation and its performance for bit loading algorithms has been compared to that achieved with M-QAM. It has been shown with an application example that in order for the number of bits per symbol obtained with M-QAM to be significantly higher than the result with M-PSK, the mean SNR must be very high. In addition, M-PSK may have some advantages over M-QAM, including PAR reduction, so the definition of SNR gap developed here is useful.

\section{ACKNOWLEDGEMENTS}

This work has been partly funded by Spanish Government with research project TIC2002-03498. The author would like to acknowledge Professor John M. Cioffi and his research group at Stanford University for their valuable help during the development of the work described in this paper. The very accurate comments of the anonymous reviewers are gratefully acknowledged.

\section{APPENDIX}

Equation (6) may be rewritten as

$$
\begin{aligned}
R & =\log _{2}\left[\left(\frac{\gamma}{\Gamma^{*}}\right)^{1 / 2}\right] \\
& =\log _{2}\left[1+\left(\frac{\sqrt{\gamma}}{\sqrt{\Gamma^{*}}}-1\right)\right] \\
& =\log _{2}\left[1+\left(\frac{\sqrt{\gamma}-\sqrt{\Gamma^{*}}}{\gamma \sqrt{\Gamma^{*}}} \gamma\right)\right] .
\end{aligned}
$$

This expression can be reformulated as:

$$
R=\log _{2}\left[1+\frac{\gamma}{\left(\frac{\sqrt{\gamma}-\sqrt{\Gamma^{*}}}{\gamma \sqrt{\Gamma^{*}}}\right)^{-1}}\right]=\log _{2}\left[1+\frac{\gamma}{\Gamma}\right]
$$

So that (1) is satisfied defining $\Gamma$ as:

$$
\begin{gathered}
\Gamma=\frac{\sqrt{\gamma \cdot \Gamma^{*}}}{1-\sqrt{\Gamma^{*} / \gamma}} . \\
\text { REFERENCES }
\end{gathered}
$$

[1] "Digital Video Broadcasting (DVB): framing structure, channel coding and modulation for digital terrestrial television," ed 1 03, ETSI ETS 300 744, 1997.

[2] "Wireless LAN Medium Access Control (MAC) and Physical Layer (PHY) specifications: high-speed physical layer extension in the 2.4 GHz band," IEEE 802.11b, 1999.

[3] R. G. Gallager, Information Theory and Realiable Communication. Wiley, 1968.

[4] T. Starr, J. M. Cioffi, and P. J. Silverman, Understanding Digital Subscriber Line Technology. Prentice Hall, 1999.

[5] P. S. Chow, J. M. Cioffi, and J. A. C. Bingham, "A practical discrete multitone transceiver loading algorithm for data transmission over spectrally shaped channels," IEEE Trans. Commun., vol. 43, no. 2/3/4, pp. 773-775, Feb/Mar/Apr 1995.

[6] R. F. H. Fischer and J. B. Huber, "A new loading algorithm for discrete multitone transmission," in Proc. IEEE Global Telecommunications Conference (GLOBECOM), vol. 1, pp. 724-278, Nov. 1996.

[7] D. Hughes-Hartogs, "Ensemble modem structure for imperfect transmission media," U.S. Patent 4833 796, May 1989.

[8] E. Baccarelli, A. Fasano, and M. Biagi, "Novel efficient bit-loading algorithms for peak-energy-limited ADSL-type multicarrier systems," IEEE Trans. Signal Processing, vol. 50, pp. 1237-1247, May 2002.

[9] W. Rhee and J. M. Cioffi, "Increase in capacity of multiuser OFDM system using dynamic subchannel allocation," in Proc. IEEE Vehicular Technology Conference (VTC), vol. 2, pp. 1085-1089, May 2000.

[10] C. Y. Wong, R. S. Cheng, K. Ben Letaief, and R. D. Murch, "Multiuser OFDM with adaptative subcarrier, bit, and power allocation," IEEE J. Select. Areas in Commun., vol. 17, no. 10, pp. 1747-1758, Oct. 1999.

[11] A. G. Armada, "A simple multiuser bit loading algorithm for multicarrier WLAN," in Proc. IEEE International Communications Conference (ICC), vol. 4, p. 1168-1171, June 2001.

[12] J. M. Cioffi, G. D. Dudevoir, M. V. Eyubouglu, and G. D. Forney Jr, "MMSE decision-feedback equalizers and coding - part II: coding results," IEEE Trans. Commun., vol. 43, no. 10, pp. 2595-2604, Oct. 1995.

[13] J. A. Davis and J. Jedwab, "Peak-to-mean power control in OFDM, Golay complementary sequences and Reed-Muller codes," IEEE Trans. Inform. Theory, vol. 45, no. 7, pp. 2397-2417, Nov. 1999.

[14] B. M. Popovic, "Synthesis of power efficient multitone signals with flat amplitude spectrum," IEEE Trans. Commun., vol. 39, no. 7, pp. 1031-1033, July 1991.

[15] J. G. Proakis, Digital Communications, 4th ed. McGraw Hill, 2000.

[16] A. G. Armada and J. M. Cioffi, "Performance of single-user and multi-user constant bit loanding in hiperlan channels," in Proc. XI European Signal Processing Conference (EUSIPCO), vol. III, pp. 187-190, Sept. 2002.

[17] S. H. Muller and J. B. Huber, "A comparison of peak power reduction schemes for OFDM," in Proc. Global Telecommunications Conference (GLOBECOM), vol. 1, pp. 1-5, Nov. 1997.

[18] M. Breiling, S. H. Muller-Weinfurtner, and J. B. Huber, "SLM peak-power reduction without explicit side information," IEEE Commun. Lett., vol. 5, no. 6, pp. 239-241, June 2001. 